\title{
Air Tightness Testing for Biosafety Level 3
}

\section{Milind Shinde*}

Manager at Elomatic Pharmalab Consulting \& Engineering, Mumbai, India

\section{Introduction}

All bio-containment guidelines and inspection documents set requirements for airtight containment boundaries around biohazards. Current international containment guidelines only scratch the surface for biosafety level 3 (BSL-3) and the available tests proposed are not entirely suitable for such facilities. Some steps in the right direction have been taken, notably as outlined in the standards put forth in the Australia-New Zealand model for their Physical Containment 3 designation.

There are several reasons why airtight containment boundaries are required, the most obvious of which is that they prevent the escape of airborne biohazards. A much less obvious function is that they also prevent the escape of the decontamination gases or vapours used. These gases and vapours are, in fact, the most likely dangerous airborne agents [1].

Key questions regarding what exactly constitutes acceptable leakage levels, however, have to be answered before quantitative testing can become the standard. Until then qualitative methods will see continued use. One of the principle requirements of BSL-3 is ensuring inward airflow into a contained area from a less contained area. This can generally be achieved by maintaining the facility under negative pressure with respect to the atmosphere, using two interlocked door airlocks at the containment boundaries and pressure cascading, etc. The effectiveness of all measures depends directly on the integrity of the room / facility. Guidance with regards BSL-3 containment barrier integrity is relatively superficially provided by the "Biosafety in Microbiological and Biomedical Laboratories" (BMBL) guidelines. According to the BMBL 'surfaces should be sealed' and 'openings should be capable of being sealed to facilitate space decontamination'. In addition, there are no criteria for verifying that these conditions have been met in the BMBL or in any official certification BSL-3 checklist.

\section{Pressure Decay Test not Suitable for BSL-3 Validation}

Methods to validate the air leakage of a containment boundary can be classified into two main categories: qualitative and quantitative. The only quantitative test prescribed in the U.S. and Canada is the Pressure Decay Test. However, the Pressure Decay Test is not well suited to BSL3 performance validation. The Pressure Decay Test is an extreme test in that it represents the highest standard for room integrity in the U.S. and Canada. It is designed for BSL-3Ag/BSL-4 containment construction validation, which is historically constructed with specialized and very expensive high performance containment barrier systems. Typical methods for constructing a BSL-3 space will not pass the Pressure Decay Test criteria and the risks associated with aerosol hazards in a BSL-3 space do not warrant constructing barriers that will pass this test either.

Without a relevant quantitative test to use, the most common methods of testing BSL-3 barriers are qualitative tests designed to find and fix holes in the barrier. That is pretty sound logic overall, but the devil is in the details and in how one records those details in the course of a construction project.

\section{Identifying Air Leakage}

The two most common methods of identifying air leaks are smoke testing and soap bubble testing. Regrettably, there is no guidance or consensus on what parameters should be used to perform these tests. The parameters have to be defined on a case by case basis via laboratory operations and failure scenarios (Figures 1 and 2).

\section{AS/NZS 2243.3:2010 Guidelines}

The only current quantitative tests in practice for BSL-3 have been developed in Australia and New Zealand for their Physical Containment 3 (PC3) designation. These tests are relatively simple to execute and do not subject containment level 3 rooms to pressures they are not designed to withstand (Level 3 rooms are tested at 0.8 " $200 \mathrm{~Pa}$ pressure difference) (Figure 3).

The standard has been developed to set acceptable leakage rates appropriate for containment level 3 that are achievable with common construction systems, i.e. drywall or sandwich panel constructions with appropriate penetration details. Careful attention to joints and penetrations is required for the rooms to pass the test. Normal workmanship for non-containment spaces will not suffice. Spaces that pass the test criteria are considered capable of gaseous fumigation and secondary containment protection.

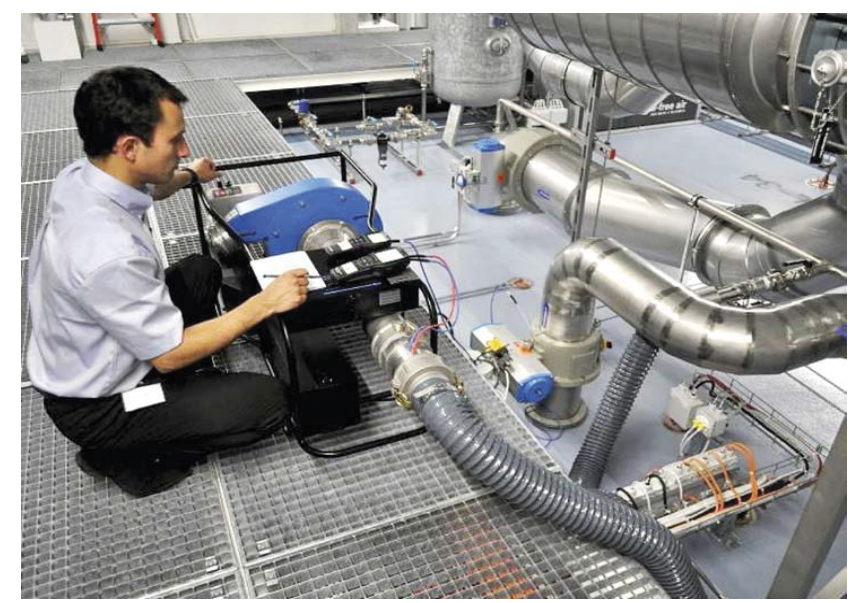

Figure 1: Air leakage fittings.

*Corresponding author: Milind Shinde, Manager at Elomatic Pharmalab Consulting \& Engineering, Mumbai, India, Tel: 91-22-25836146; E-mail: milind.shinde@elomatic.com

Received July 10, 2014; Accepted August 04, 2014; Published August 12 , 2014

Citation: Shinde M (2014) Air Tightness Testing for Biosafety Level 3. J Bioprocess Biotech 4: 173 doi: 10.4172/2155-9821.1000173

Copyright: $\odot 2014$ Shinde M. This is an open-access article distributed under the terms of the Creative Commons Attribution License, which permits unrestricted use, distribution, and reproduction in any medium, provided the original author and source are credited. 


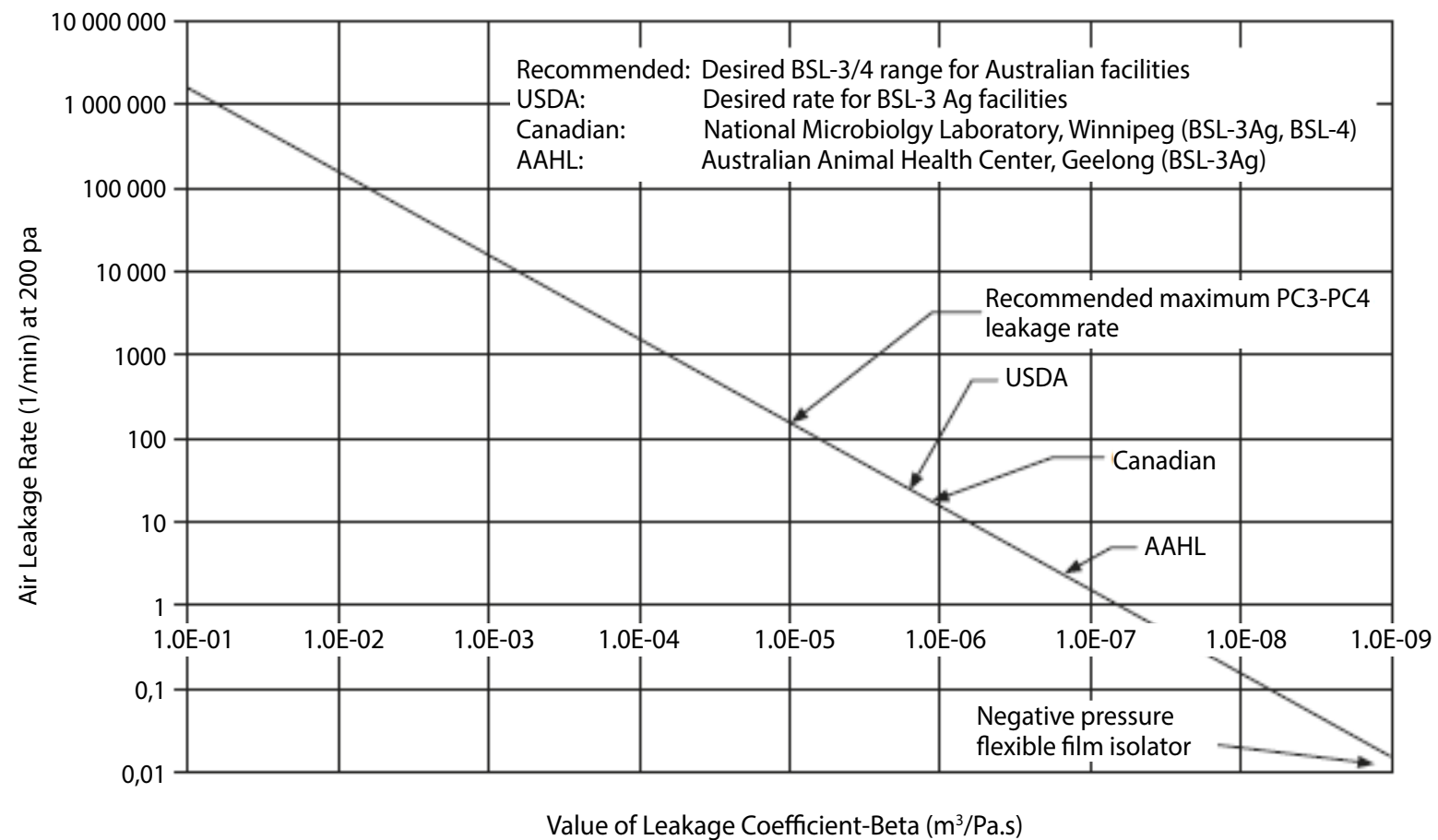

Figure 2: Graph explaining value of leakage coefficient Vs air leakage rate.

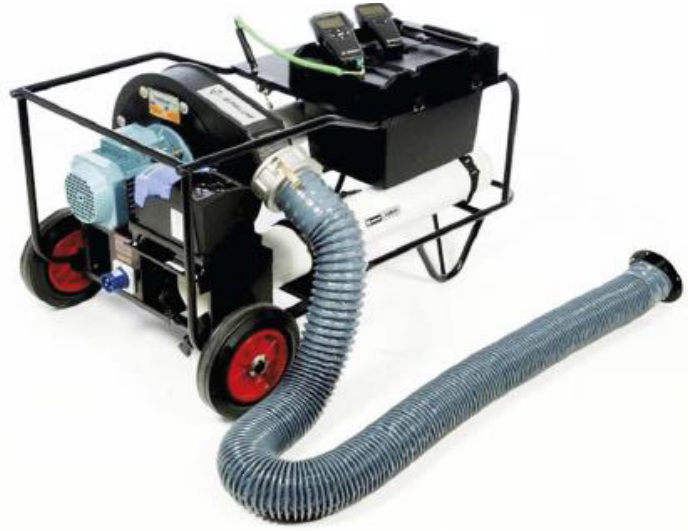

Figure 3: Air suppliers

Details regarding tests for leak tightness (BSL-3Ag and BSL-3) and the room leak tightness test (Pressure Decay test BSL-3AG) are provided in segments.

\section{Segment 1}

The basic procedure for room air leakage testing under negative pressure for BSL-3Ag and BSL-3 according to AS/NZS 2243.3:2010 is as follows:

Air leakage can be quantified by using an equilibrium pressure/ flow test. For smaller rooms a vacuum cleaner connected to the supply or exhaust duct by a hose and a control valve can be used. For larger rooms a blower may be required.

1. This test usually involves the introduction of clean, dry compressed air into the space while monitoring the pressure in the space through separate pressure tapping (a vacuum cleaner with a hose and integrated anemometer could be used for low leakage rates).

2. When the pressure is stabilized at the required test pressure (200 $\mathrm{Pa}$ ), the inflow of air required to maintain this pressure is measured using a flow meter such as a variable gap meter.

3. The leakage is then recorded in litres per minute.

4. Prior to the test care needs to be taken to ensure that all sources of air or gas pressure within the space are isolated. Doors should be taped with PVC tape and physically restrained to prevent movement under the positive room pressure.

This test can also be performed by extracting air from the room, thus placing the room under negative pressure.

Acceptance criteria Leakage must be $<20$ litres per minute for BSL $3 \mathrm{Ag}$, and $<200$ litres per minute for BSL 3 . All instruments should be appropriately calibrated by an accredited laboratory.

\section{Segment 2}

For pretesting, an initial pressure of 125 to 250 8ontainment barrier so that it is not affected by air distribution. The minimum accuracy of the manometer should be $10 \mathrm{~Pa}$ (0.05 in. w.g.) and capable of reading pressure up to $750 \mathrm{~Pa}$.

3. Install a ball valve in the piping between the vacuum pump/fan and the room to allow the room to be sealed once the test pressure has been attained.

4. Connect a vacuum source to the room and create a $500 \mathrm{~Pa}$ 
negative pressure differential; allow the room to stabilize and close the valve between the vacuum pump/ fan and the room to seal room at $500 \mathrm{~Pa}$.

5. Dynamically trend pressure loss starting at $500 \mathrm{~Pa}$ negative pressure differentials; record the differential pressure at 1 minute intervals for 20 minutes.

6. If repeat test is required, allow for a 20 minute waiting period.

7. Disconnect the vacuum pump/fan and open the ball valve slowly to allow room pressure to return to normal.

Acceptance criteria Two consecutive tests with a minimum $250 \mathrm{~Pa}$ (1 in.w.g.) loss of pressure from an initial $500 \mathrm{~Pa}(2$ in. w.g.) over a 20 minute period. All instruments should be appropriately calibrated by an accredited laboratory.

\section{Practical Difficulties and Limitations of Current Testing Methods}

Acceptance criteria for all leakage testing methods are defined for single rooms with sturdy constructions, a limited number of penetrations (for ducting, electrical cables, doors, view panels etc.) and airtight view panels as well as airtight doors.

It is feasible to qualify of single room laboratory or one animal room with airtight doors, but if you are testing a BSL- 3 laboratory facility that consists of several laboratory rooms connected with a BSL3 corridor, the laboratory door is not airtight. The doors on a BSL-3 containment barrier are airtight, but internal doors normally leak. While testing for leakage, single rooms with leaking doors need to be qualified. The acceptance criteria cannot be met unless the non-airtight door/doors is/are sealed with adhesive tape or sealant.

For large rooms with multiple doors, large surface areas and several penetrations it is difficult to fulfil the acceptance criteria. It is necessary to define the acceptance criteria in terms of litres/min per square meters of the room's surface area [2].

In pressure decay testing the test is sensitive to changes in the ambient temperature during the course of the test. Therefore, an appropriate correction factor has to be applied that considers the temperature before and after the test.

The Australia-New Zealand model (AS/NZS 2243.3:2010) provides a common approach and platform for designers, builders, and owners to engage, deliver, and qualify the facility. The standard is meant to ensure that containment laboratories are designed and constructed to perform their functions safely and consistently, to a standard that is deemed appropriate and fit for the purpose by an independent body.

The Australia-New Zealand model can be improved upon with a more evidence-based rationale for performance requirements. What level of air leakage at a given pressure correlates to a safe measure of containing hazardous gases in likely scenarios? Is the acceptable leakage amount dependent on room volume or the same for all room sizes? Answering these questions, as well as identifying the relationship of airtightness to the reduction in risk of airborne pathogen release, even in a qualified way, would take us a long way towards setting a standard that is rational and defendable and which will satisfy industrial, regulatory, and public interests.

Until that exists, we can do what is reasonable to ensure sound and appropriate laboratories, which I believe means qualitative testing with well-considered test protocols based on what the laboratory is intended for. As we perform these tests and share the outcomes we will build a common approach and standard that will begin to standardize this important containment protective measure.

\section{References}

1. https://law.resource.org/pub/nz/ibr/as-nzs.2243.3.2010.pdf

2. http://www.cdc.gov/biosafety/publications/bmbl5/bmbl.pdf 